\title{
Covariance of regenerative mean and variance estimators for the Markov chains
}

\author{
James M. Calvin \\ Department of Operations Research \\ Stanford University \\ Stanford, California 94305
}

\section{ABSTRACT}

This paper explores the asymptotic covariance structure of the mean and standard deviation estimators used in the regenerative method of simulation output analysis. It is shown that the asymptotic covariance of the mean and standard deviation estimators does not depend on the choice of return state.

\section{INTRODUCTION}

The regenerative method of simulation output analysis uses the fact that the interblocks of a regenerative stochastic process are independent and identically distributed to construct a consistent estimator of the variance constant used to derive confidence intervals. If a process has more than one regeneration point, the estimator will have the same limiting value no matter which point is used to block the observations. While all such estimators have the same limit, different regeneration points may yield more or less variable variance estimators. A common rule of thumb for obtaining an estimator with low variance is to choose the regeneration point that has the least mean regeneration time.

Glynn and Iglehart (1987) proved a bivariate central limit theorem for the regenerative point estimator and the standard deviation estimator. Numerical calculations presented in the paper showed that the off-diagonal element in the covariance matrix appeared to be independent of the return state used to delimit regenerative cycles. The purpose of this paper is to derive an expression for the covariance matrix in the case of a class of Markov chains. The expressions derived show that the off-diagonal term is independent of the return state. Some insight is gained into the nature of the variance of the variance estimators for different return states. An example is given where the state that yields the least variable variance estimator has the greatest mean regeneration time.

The next section introduces the notation. Section 3 presents two lemmas that are used in section 4 to provide a simple expression for the covariance matrix. Section 5 contains a simple example of a chain with three states.

\section{NOTATION}

Let $\left\{X_{n} ; n=0,1,2, \cdots\right\}$ be a Markov chain with state space $S$, where $S$ is a finite set or a subset of a Euclidean space. The probability law of the chain corresponding to initial probability distribution $\varphi$ will be denoted $P_{\varphi}$. We write $E_{\varphi}$ for the expectation with respect to the probability $P_{\varphi}$, and if $\varphi$ is degenerate at $x$, then we write $P_{x}$ and $E_{x}$.

For a subset $A$ of $S_{1} s_{A}$ will denote the first return time of the chain to $\mathrm{A}$ and $\tau_{A}$ will denote tlie first hitting time of the set $\mathrm{A}$ ( $\mathrm{s}$ and $\tau$ coincide unless the chain starts in $A$, in which case $s_{A}=0$ ). We will write $s_{x}$ and $\tau_{x}$ instead of $s_{\{x\}}$ and $\tau_{\{x\}}$.

We will consider only clains that are unformly $\varphi$-recurrent (Orey (1971)); that is, there exists a $\sigma$-finite measure $\varphi$ such that if $\varphi(A)>0$, then $P_{x}\left[\tau_{A} \geq n\right] \rightarrow 0$ uniformly in $x \in S$. Uniformly $\varphi$-recurrent chains have a unique invariant probability measure, which will be denoted by $\pi$. We assume that the chain is aperiodic.

Throughout the paper $z$ will denote a fixed return state with $\pi(\{z\})>$ 0 (so that $E_{z}\left(\tau_{z}\right)<\infty$ ). When subscripts are omitted the state will be understood to be $z$ : e.g. $E(\tau)=E_{z}\left(r_{z}\right)$.

Let $\mathrm{f}$ be a bounded real valued function on $\mathrm{S}$, with $E_{\pi} f(X)=0$.

\section{MOMENT CALCULATIONS}

Let $\tau$ and $Z$ be a random variables that have the same distributions as $\tau_{z}$ and

$$
\sum_{n=1}^{\tau_{z}} f\left(X_{n}\right)
$$

respectively, under $P_{z}$. In this section we will derive expressions for moments of the form $E\left(\tau^{i} Z^{j}\right)$; these moments will be used in the next section to describe the covariance matrix. We start by defining several quantities that depend on the transition probability of the chain, but not on the return state $z$. Let

$$
\begin{gathered}
\sigma^{2} \triangleq E_{\pi} f\left(X_{0}\right)^{2}+2 \sum_{n=1}^{\infty} E_{\pi}\left[f\left(X_{0}\right) f\left(X_{n}\right)\right] \\
m_{3}=E_{x} f\left(X_{0}\right)^{3}+3 \sum_{n=1}^{\infty} E_{\pi}\left[f\left(X_{0}\right)^{2} f\left(X_{n}\right)\right]+3 \sum_{n=1}^{\infty} E_{\pi}\left[f\left(X_{0}\right) f\left(X_{n}\right)^{2}\right] \\
+6 \sum_{n=1}^{\infty} \sum_{m=1}^{\infty} E_{\pi}\left[f\left(X_{0}\right) f\left(X_{n}\right) f\left(X_{n+m}\right)\right]
\end{gathered}
$$

and

$$
\begin{gathered}
m_{4}=E_{\pi} f\left(X_{0}\right)^{4}+4 \sum_{n=1}^{\infty} E_{\pi}\left[f\left(X_{0}\right)^{3} f\left(X_{n}\right)\right]+4 \sum_{n=1}^{\infty} E_{\pi}\left[f\left(X_{0}\right) f\left(X_{n}\right)^{3}\right] \\
+6 \sum_{n=1}^{\infty}\left\{E_{\pi}\left[f\left(X_{0}\right)^{2} f\left(X_{n}\right)^{2}\right]-E_{\pi}\left[f\left(X_{0}\right)^{2}\right] E_{\pi}\left[f\left(X_{n}\right)^{2}\right]\right\} \\
+12 \sum_{n=1}^{\infty} \sum_{m=1}^{\infty}\left\{E_{\pi}\left[f\left(X_{0}\right)^{2} f\left(X_{n}\right) f\left(X_{n+m}\right)\right]-\right. \\
+12 \sum_{n=1}^{\infty} \sum_{m=1}^{\infty} E_{\pi}\left[f\left(X_{0}\right) f\left(X_{n}\right)^{2} f\left(X_{n+m}\right)\right] \\
+12 \sum_{n=1}^{\infty} \sum_{m=1}^{\infty} E_{\pi}\left[f\left(X_{0}\right) f\left(X_{n}\right) f\left(X_{n+m}\right)^{2}\right]
\end{gathered}
$$


$+24 \sum_{n=1}^{\infty} \sum_{m=1}^{\infty} \sum_{k=1}^{\infty} E_{\pi}\left[f\left(X_{0}\right) f\left(X_{n}\right) f\left(X_{n+m}\right) f\left(X_{n+m+k}\right)\right]$.

We will express moments of the form $E\left(\tau^{i} Z^{j}\right)$ in terms of the above constants and also the following quantities that do in general depend on the choice of return state $z$ :

$$
\begin{gathered}
\chi_{1}=E_{x}\left[f(X) E_{X}\left(s_{z}\right)\right], \\
\chi_{2}=E_{\pi}\left[f^{2}(X) E_{X}\left(s_{z}\right)\right]+2 \sum_{n=1}^{\infty} E_{x}\left[f\left(X_{0}\right) f\left(X_{n}\right) E_{X_{n}}\left(s_{z}\right)\right], \\
\eta_{1}=\sum_{n=1}^{\infty}\left\{E_{\pi}\left[f\left(X_{n}\right)\right]-E_{z}\left[f\left(X_{n}\right)\right]\right\}=-\sum_{n=1}^{\infty} E_{z}\left[f\left(X_{n}\right)\right]
\end{gathered}
$$

and

$$
\eta_{2}=\sum_{n=1}^{\infty}\left[\sigma^{2}-\sigma_{z, n}^{2}\right]
$$

where

$$
\sigma_{z, n}^{2}=E_{z}\left[f\left(X_{n}\right)^{2}\right]+2 \sum_{m=1}^{\infty} E_{z}\left[f\left(X_{n}\right) f\left(X_{n+m}\right)\right]
$$

The following two lemmas can be proved using methods similar to those in Chung (1967); in particular, Chung's Theorem I.14.7.

Lemma 1: If

$$
E_{z}\left(r_{z} \sum_{n=1}^{\tau_{z}}\left|f\left(X_{n}\right)\right|\right)<\infty
$$

then

$$
E[\tau Z]=E(\tau)\left(\chi_{1}+\eta_{1}\right)
$$

Also

$$
\begin{aligned}
E\left[\tau Z^{2}\right]= & \frac{1}{2} \sigma^{2}\left[E(\tau)+E\left(\tau^{2}\right)\right]+2 E(\tau) \sum_{n=1}^{\infty} n E_{\pi}\left[f\left(X_{0}\right) f\left(X_{n}\right)\right] \\
& +E(\tau)\left(\left(\chi_{1}+\eta_{1}\right)^{2}+\chi_{1}^{2}+\eta_{1}^{2}+\chi_{2}+\eta_{2}\right) .
\end{aligned}
$$

Lemma 2: If the series in the definitions of $m_{2}, m_{3}$, and $m_{4}$ converge absolutely, then

$$
\begin{gathered}
E\left(Z^{2}\right)=E(\tau) m_{2} \\
E\left(Z^{3}\right)=E(\tau)\left[m_{3}+3 m_{2}\left(\chi_{1}+\eta_{1}\right)\right]
\end{gathered}
$$

and

$$
\begin{aligned}
& E\left(Z^{4}\right)= \\
& E(\tau)\left(m_{4}+4 m_{3}\left(\chi_{1}+\eta_{1}\right)+6 m_{2}\left(\left(\chi_{1}+\eta_{1}\right)^{2}+\chi_{1}^{2}+\eta_{1}^{2}+\chi_{2}+\eta_{2}\right)\right) .
\end{aligned}
$$

\section{ESTIMATOR COVARIANCE MATRIX}

Let $S_{0}=0$, and $S_{n}=f\left(X_{0}\right)+\cdots+f\left(X_{n-1}\right)$. Under the assumptions on the chain described in section 2 ,

$$
\frac{1}{n} E\left(S_{n}^{2}\right) \rightarrow \sigma^{2}
$$

as $n \rightarrow \infty$, and

$$
n^{-1 / 2} S_{n} \Rightarrow \mathcal{N}\left(0, \sigma^{2}\right)
$$

as $n \rightarrow \infty$. We are interested in estimating $\sigma^{2}$ in order to obtain confidence intervals. In the regenerative method, $S_{n}$ is divided up into independent blocks by starting a new block whenever a regeneration point is reached. If $Z_{i}$ is the $i t h$ block, then

$$
\sigma^{2}=\lim _{n \rightarrow \infty} \frac{1}{n} E\left(Z_{1}+\cdots+Z_{K_{n}}\right)^{2}=\lim _{n \rightarrow \infty} \frac{1}{n} \sum_{j=1}^{K_{n}} E\left(Z_{j}^{2}\right)
$$

as $n \rightarrow \infty$, where $K_{n}$ is the number of regenerations in $n$ observations. Choosing different regeneration points will in general give different estimator variances.

Let $r(n)$ and $s(n)$ denote the regenerative mean and standard cleviation estimators, respectively, based on observation of the chain up to time $n$. It is shown in Glynn and Iglehart (1987) (for general regenerative processes) that

$$
n^{1 / 2}(r(n), s(n)-\sigma) \Rightarrow(r, s) \sim \mathcal{N}(0, D),
$$

where

$$
\begin{gathered}
D_{11}=E\left(Z^{2}\right) / E(\tau), \\
D_{12}=\frac{E\left(Z^{3}\right)-3 \sigma^{2} E(\tau Z)}{2 \sigma E(\tau)},
\end{gathered}
$$

and

$$
\begin{gathered}
D_{22}=\left(E\left(Z^{4}\right)-2 \sigma^{2} E\left(\tau Z^{2}\right)+\sigma^{4} E\left(r^{2}\right)\right) /\left(4 \sigma^{2} E(\tau)\right) \\
-\left(4 E(\tau Z) E\left(Z^{3}\right)-8 \sigma^{2}[E(\tau Z)]^{2}\right) /\left(4 \sigma^{2} E(\tau)^{2}\right) .
\end{gathered}
$$

Using the formulas from the lemmas, the covariance matrix can be written

$$
D=\left[\begin{array}{cc}
\sigma^{2} & \frac{m_{3}}{2 \sigma} \\
& \\
& c+\chi_{1}^{2}+\eta_{1}^{2} \\
\frac{m_{3}}{2 \sigma} & +\chi_{2}+\eta_{2}
\end{array}\right],
$$

where

$$
c \triangleq \frac{m_{4}}{4 \sigma^{2}}-\frac{\sigma^{2}}{4}-\sum_{n=1}^{\infty} n E_{n}\left[f\left(X_{0}\right) f\left(X_{n}\right)\right]
$$

is independent of the return state. The first term in the expression for $c$ is proportional to the (limiting) coefficient of kurtosis of the random variable $S_{n}$. Thus if $S_{n}$ has large kurtosis, the standard deviation estimator will be highly variable no matter which return state is used. Notice that the diagonal term is also independent of the return state $z$, since as previously mentioned, $\sigma^{2}$ and $m_{3}$ are independent of the return state.

Let $\kappa_{n}$ be the coefficient of skewness for the random variable $S_{n}$ under the initial distribution $\pi$;

$$
\kappa_{n}=\frac{E_{\pi}\left(S_{n}^{3}\right)}{E_{\pi}\left(S_{n}^{2}\right)^{3 / 2}}
$$

Clearly $\kappa_{n}$ is defined independently of the return state, and

$$
\kappa \triangleq \lim _{n \rightarrow \infty} \kappa_{n}=\frac{m_{3}}{\sigma^{3}}
$$

With this nolation, $D_{12}=\frac{1}{2} \sigma^{2} \kappa$. For any symmetrical chain, for example a birth and death process on $\{-N, \cdots, 0, \cdots, N\}$ for which the birth and death parameters as well as the values of the function $f$ are symmetrical about $0, k=0$ and so $r$ and $s$ are orthogonal.

Choosing a return state to minimize variance of the standard deviation estimator is equivalent to choosing a return state to maximize correlation between the estimators for the mean and the standard deviation. 


\section{EXAMPLE}

Consider the Markov chain with state space $S=\{1,2,3\}$ and transition matrix

$$
P=\left[\begin{array}{ccc}
1-\epsilon & \epsilon & 0 \\
1 / 2 & 0 & 1 / 2 \\
0 & \epsilon & 1-\epsilon
\end{array}\right],
$$

for $0<\epsilon<1$. The stationary distribution is

$$
\pi=\left(\frac{1}{2+2 \epsilon}, \frac{2 \epsilon}{2+2 \epsilon}, \frac{1}{2+2 \epsilon}\right) .
$$

Let $f=(-M, 0, M)$ for some $M>0$, so that $E_{\pi} f=0$. The values of the quantities that vary with state are given below (the values for state 3 are the same as for state 1 ).

$\begin{array}{lllll}\text { State } & \eta_{1}^{2} & \chi_{1}^{2} & \eta_{2} & \chi_{2} \\ 1,3 & \frac{M^{2}}{\xi^{2}} & \frac{M^{2}(1-\epsilon)^{2}}{\epsilon^{2}} & \frac{M^{2}(2-\epsilon)}{\epsilon^{2}} & \frac{M^{2} \epsilon(2-\epsilon)}{(1+\epsilon)^{2}} \\ 2 & 0 & 0 & \frac{M^{2}(2-\epsilon)}{\varepsilon^{2}(1+\varepsilon)} & -\frac{M^{2}(2-\epsilon)}{(1+\varepsilon)^{2}}\end{array}$

The difference in variances is

$$
D_{22}(1)-D_{22}(2)=2\left(\frac{M}{\epsilon}\right)^{2} \text {, }
$$

while

$$
E_{1}\left(\tau_{1}\right)=E_{3}\left(\tau_{3}\right)=2+2 \epsilon \rightarrow 2, \quad E_{2}\left(\tau_{2}\right)=\frac{1+\epsilon}{\epsilon} \rightarrow \infty
$$

as $\epsilon \downarrow 0$. Therefore, while the mean regeneration time for state 2 grows without bound as $\epsilon \downarrow 0$, it gives the least variable estimator, with the difference going to $\infty$ as $\varepsilon \downarrow 0$. Essentially all of the difference is accounted for by the $\eta_{1}^{2}$ and $\chi_{1}^{2}$ terms.

In this example, the kurtosis of $S_{n}$ increases as $M$ increases or $\epsilon$ decreases, so the variance of all 3 standard deviation estimators increases as $\epsilon \downarrow 0$. Since the chain is symmetrical, $\kappa=0$ and the mean and standard deviation estimators are asymptotically independent.

\section{CONCLUSION}

The covariance matrix that appears in the central limit theorem for the regenerative mean and standard deviation estimators has been expressed in a form so that several conclusions could be reached. The offdiagonal term, representing the covariance between the point and standard deviation estimators, is independent of the return state chosen for blocking. The expression for the variance of the standard deviation estimator shows that the variance is increased by kurtosis in the partial sum random variables. The variance does depend on the return state used for blocking, and an example showed that the state with the shortest mean return time can have the greatest variance.

\section{REFERENCES}

Chung, K.L. (1967). Markov Chains with Stationary Transition Probabilities, Second Edition. Springer-Verlag, Berlin-New York-Heidelberg.

Glynn, P.W. and D.L. Iglehart. (1987). A Joint Central Limit Theorem for the Sample Mean and Regenerative Variance Estimator. Annals of Operations Research 8, 41-55.

Orey, S. (1971). Lecture Notes on the Limit Theorems for Markov Chain Transition Probabilities. Van Nostrand, New York.

\section{AUTHOR'S BIOGRAPHY}

JAMES M. GALVIN is a graduate student in the department of Operations Research at Stanford University. His interests are in applied probability and optimization.

James M. Calvin

Department of Operations Research

Stanford University

Stanford, California 94305

(415)-723-9383 